\title{
Direct thrust measurement of a permanent magnet helicon double layer thruster
}

\author{
K. Takahashi, ${ }^{1, a)}$ T. Lafleur, ${ }^{1}$ C. Charles, ${ }^{1, b)}$ P. Alexander, ${ }^{1}$ R. W. Boswell, ${ }^{1}$ M. Perren, ${ }^{2}$ \\ R. Laine, ${ }^{2}$ S. Pottinger, ${ }^{3}$ V. Lappas, ${ }^{3}$ T. Harle, ${ }^{3}$ and D. Lamprou ${ }^{3}$ \\ ${ }^{1}$ Space Plasma, Power and Propulsion Group, Research School of Physics and Engineering, \\ The Australian National University, Canberra ACT 0200, Australia \\ ${ }^{2}$ ASTRIUM-EADS, 6 rue Laurent Pichat, 75016 Paris, France \\ ${ }^{3}$ Surrey Space Centre, University of Surrey, Guildford GU2 7XH, United Kingdom
}

(Received 24 February 2011; accepted 22 March 2011; published online 7 April 2011)

\begin{abstract}
Direct thrust measurements of a permanent magnet helicon double layer thruster have been made using a pendulum thrust balance and a high sensitivity laser displacement sensor. At the low pressures used $(0.08 \mathrm{~Pa})$ an ion beam is detected downstream of the thruster exit, and a maximum thrust force of about $3 \mathrm{mN}$ is measured for argon with an rf input power of about $700 \mathrm{~W}$. The measured thrust is proportional to the upstream plasma density and is in good agreement with the theoretical thrust based on the maximum upstream electron pressure. () 2011 American Institute of Physics. [doi:10.1063/1.3577608]
\end{abstract}

The recent discovery of current-free double layers (DLs) in rf plasmas, ${ }^{1,2}$ together with the formation of ion beams in such systems, ${ }^{2}$ has stimulated a renewed interest in plasma expansion studies, especially within a propulsion context, ${ }^{3-5}$ since such systems can, in principle, be used to provide thrust for spacecraft. In contrast to more familiar electrostatic acceleration thrusters, such as ion engines and hall thrusters, ${ }^{6}$ plasma thrusters make use of ambipolar electric fields or DLs to accelerate ions to supersonic speeds. ${ }^{1,3}$ These systems typically consist of an insulating source tube and a magnetic field $^{3,5,7-9}$ used to provide plasma confinement and produce plasma expansion, hence forming the DL. ${ }^{3,10}$ An rf antenna is used to produce the plasma, which is usually inductively ${ }^{11}$ or wave coupled. ${ }^{7,9}$ Since the ambipolar fields/DLs are internal structures within the plasma, no biased electrodes are needed, hence reducing erosion of system components. ${ }^{3}$ In addition, the results of a number of studies suggest that sufficient electrons escape the DL to neutralize the downstream ion beam. ${ }^{12}$ Thus a separate neutralizer system is not needed.

Experimental research on plasma thrusters has to date involved studies of the fundamental physics associated with $\mathrm{DLs},{ }^{2,3}$ the use of different propellant gases, ${ }^{3,13}$ helicon wave characterization, ${ }^{8}$ and the use of permanent magnets. ${ }^{5,9}$ Performance estimations with regard to propulsion applications are only now starting to be considered. ${ }^{7}$ Initial theoretical and thruster performance modeling has been performed, ${ }^{4}$ notably by Fruchtman, ${ }^{14,15}$ but these predictions have not yet been tested experimentally due to the lack of explicit experimental thrust measurements. In this letter we present thrust measurements of a permanent magnet helicon DL thruster (PM-HDLT), and analyze the results in the context of some of these recent models.

The PM-HDLT (custom built at Iwate University), a schematic of which is shown in Fig. 1, has been described previously. ${ }^{5}$ It consists of a $6.4 \mathrm{~cm}$ diameter Pyrex source

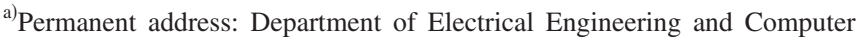
Science, Iwate University, Morioka 020-8551, Japan. Electronic mail: kazunori@iwate-u.ac.jp.

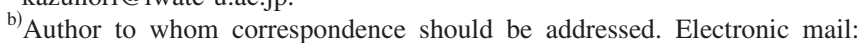
christine.charles@anu.edu.au.
}

tube (terminated at one end by an insulating plate), surrounded by a double-turn loop antenna operated at 13.56 $\mathrm{MHz}$, and supplied with between 0-900 W connected through a $\pi$-type matching network. Surrounding the source tube and antenna are a combination of two concentric neodymium iron boron $(\mathrm{NdFeB})$ permanent magnet arrays, which produce a magnetic field of about $20 \mathrm{mT}$ within the source region, decreasing to a few millitesla within the downstream region $(z>0 \mathrm{~cm}$; see Fig. 1).

The PM-HDLT is placed within the larger Irukandji space simulation chamber (custom built at the Australian National University; not shown in Fig. 1) also previously described. ${ }^{11}$ This chamber is $1 \mathrm{~m}$ in diameter and $1.4 \mathrm{~m}$ long and is pumped down to a base pressure of around $0.1 \mathrm{mPa}$ with a turbomolecular/rotary pump system. Argon gas is fed into the PM-HDLT via a small ceramic tube that passes through the insulating plate, and the working pressure is

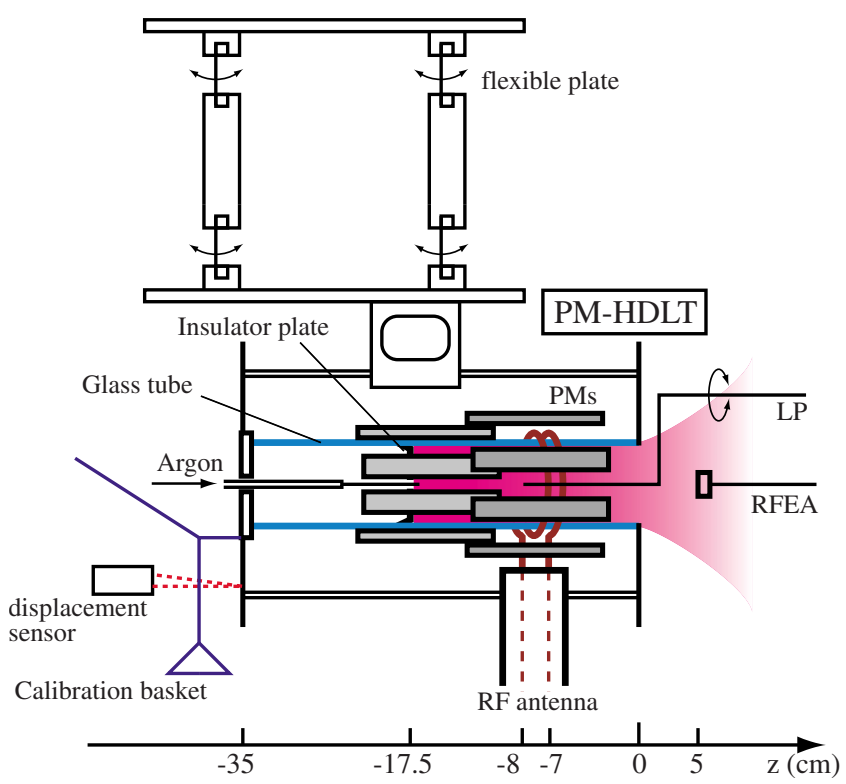

FIG. 1. (Color online) Schematic of the PM-HDLT attached to the thrust balance. Also shown are the laser displacement sensor, thrust balance calibration system, rf antenna, and diagnostic probes. 
measured with a baratron gauge. For the current investigation, a constant flow rate of $0.6 \mathrm{mg} \mathrm{s}^{-1}$ (giving a pressure of about $0.08 \mathrm{~Pa}$ ) is used.

The PM-HDLT source is mounted onto a grounded thrust balance system (custom built at the Surrey Space Centre). The thrust balance consists of four aluminum support columns (not shown in Fig. 1) onto which a double pendulum system is attached. The pendulum makes use of $0.1 \mathrm{~mm}$ thick stainless steel flexible plates, and the PM-HDLT attaches to the bottom of the pendulum as illustrated in Fig. 1 . Both the gas feedthrough and rf antenna are not in physical contact with the PM-HDLT source tube. The displacement of the pendulum is measured using a high sensitivity rf-shielded Micro-Epsilon laser displacement sensor, which is mounted to the aluminum support structure of the thrust balance.

Forces applied to the thrust stand result in an oscillation of the pendulum with a frequency of about $1 \mathrm{~Hz}$. To analyze the data, the laser system is run with no plasma to establish a baseline equilibrium position for $20 \mathrm{~s}$, then the plasma is turned on and data taken for a further $10 \mathrm{~s}$, following which the plasma is turned off and another set of data taken for a $20 \mathrm{~s}$. The data are then filtered to remove low-frequency oscillations, and the displacement is then established. To calibrate the thrust stand a small mass piece is tied to the back of the source tube with a thin horizontal thread, and a second thread attached to the support structure of the thrust stand (see Fig. 1). In this way the applied mass is in static equilibrium, and from simple mechanics the force applied to the stand can be calculated. The sensitivity was found to be about $340 \mathrm{mN} / \mathrm{mm}$ with a resolution of $0.034 \mathrm{mN}$.

Finally, a retarding field energy analyzer (RFEA) with aperture facing the source region ${ }^{2}$ is used to measure the downstream ion energy distribution function (IEDF) and hence detect the presence of an ion beam downstream of the PM-HDLT, while a $4 \mathrm{~mm}$ disk Langmuir probe (LP) is used to measure the upstream plasma density. The plasma density is found from the ion saturation region of the measured $I V$ curve, and Sheridan's method ${ }^{16}$ is used to account for expansion of the sheath around the LP disk.

Figure 2 shows thrust measurements (open squares) obtained with the thrust balance and laser displacement sensor as a function of effective rf power. The effective power is the actual power absorbed by the plasma and is found by taking resistance measurements within the matching network to establish the power transfer efficiency. ${ }^{17}$ The thrust begins quite low at less than $0.5 \mathrm{mN}$ for $140 \mathrm{~W}$, before increasing approximately linearly with effective power to almost $3 \mathrm{mN}$ for $725 \mathrm{~W}$.

The density within the source region at $z=-8 \mathrm{~cm}$ is shown as the crosses in Fig. 2. Here, the density is seen to increase approximately linearly with effective rf power, going from around $3.5 \times 10^{17} \mathrm{~m}^{-3}$ at $140 \mathrm{~W}$ to about 1 $\times 10^{18} \mathrm{~m}^{-3}$ at $725 \mathrm{~W}$ (the increase is not directly proportional to the power, due to possible neutral depletion effects that begin to occur at these higher power levels).

During operation of the PM-HDLT, an ion beam is detected in the downstream region and is associated with the presence of a DL previously measured near the exit of the thruster. $^{5,18}$ The IEDFs at $z=5 \mathrm{~cm}$ are shown in Fig. 3 for a number of effective rf powers. The IEDFs show a large lowenergy peak centered at around $50 \mathrm{~V}$ (almost independent of effective power), and a high energy ion population. The experimental IEDF for $725 \mathrm{~W}$ (open circles) has been fitted

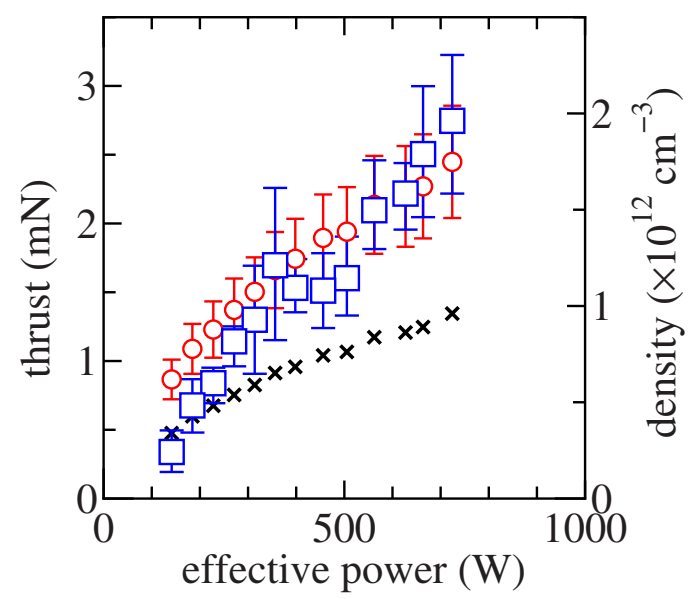

FIG. 2. (Color online) Thrust as a function of effective rf power for an argon flow rate of $0.6 \mathrm{mg} \mathrm{s}^{-1}(0.08 \mathrm{~Pa})$. The open squares show the experimental measurements while the open circles show the thrust calculated from Eq. (1). The experimental error bars show an estimate of the uncertainty in the measurements based on the analysis of the data while the theoretical error bars result from the uncertainty in the electron temperature used in Eq. (1) The crosses show the upstream plasma density on axis at $z=-8 \mathrm{~cm}$ as a function of effective rf power.

with two Gaussian ion populations, one representing the background ions, and the other the beam ions (dashed curves). ${ }^{2}$ For this power the ion beam energy is about $30 \mathrm{eV}$ higher than the local ion population.

Fruchtman ${ }^{15}$ has recently performed a theoretical analysis of the thrust expected from a plasma thruster such as the type presented here. Using a radially averaged plasma, and ignoring collisions and the effect of expansion due to a magnetic field, the thrust is given by the maximum electron pressure within the source region as

$$
T=q n_{0} A T_{e},
$$

where $q$ is the electron charge magnitude, $n_{0}$ is the maximum radially averaged density within the source region, $A$ is the cross-sectional area of the source tube, and $T_{e}$ is the electron temperature. Since no electrodes are present within the plasma, the plasma flow momentum is conserved, and momentum is transferred from the electron pressure into di-

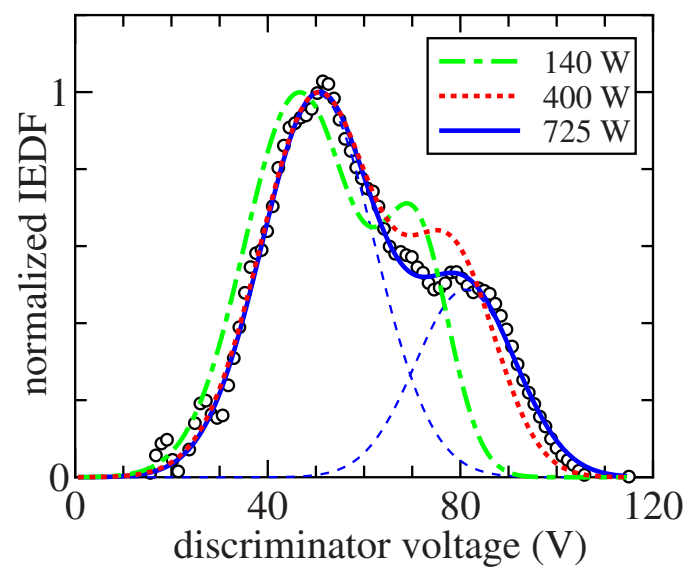

FIG. 3. (Color online) IEDFs as a function of effective rf power taken with the RFEA located at $z=5 \mathrm{~cm}$. The open circles show the experimental IEDF for $725 \mathrm{~W}$ while the solid line shows the sum of the Gaussians (dashed lines) used in the deconvolution. The dotted-dashed and dotted lines represent the sum of the fitted Gaussians for effective powers of $140 \mathrm{~W}$ and 400 $\mathrm{W}$, respectively. 
rected ion momentum ${ }^{15}$ through the DL, resulting in ion acceleration as seen by the higher energy ion population in the IEDFs shown in Fig. 3. Using the upstream plasma densities from Fig. 2 , and a scaling factor $(\approx 0.82)$ obtained from a measurement of the radial density profile, Eq. (1) is plotted in Fig. 2 (open circles). Here an electron temperature of $6 \mathrm{eV} \pm 1 \mathrm{eV}$ has been used based on measurements with the LP, and application of a particle balance ${ }^{19}$ in the thruster source region. As is seen, the theoretical thrust is in very good agreement with that measured using the thrust balance, illustrating that the thrust is directly proportional to the plasma density. Fruchtman ${ }^{14}$ has also performed a theoretical analysis of the thrust from a system with an expanding magnetic field and found that the field effectively increases the flow area of the thruster, so that the thrust increases with axial distance. In the present case, however, the plasma density rapidly decays past $z=0 \mathrm{~cm}$, and the contribution to the thrust from this magnetic expansion term is estimated to be small.

DLs cannot increase the net plasma momentum, ${ }^{14}$ and thus from Eq. (1), the presence of a DL can only affect the thrust through changes in the upstream plasma density or electron temperature. Since the spontaneous formation of a DL is inherent to the present geometry and magnetic field configuration, it is not possible to compare the results with a non-DL condition under similar operating conditions. For the calculated thrust from Eq. (1), the electron temperature was estimated from LP measurements and separately from a particle balance. Since the results match those of the thrust measurements, this suggests that under the current operating conditions the DL does not significantly change the upstream electron temperature.

While the measured thrust in Fig. 2 is quite low for the applied power when compared with existing propulsion systems of similar powers, ${ }^{6}$ it should be said that the PM-HDLT is an unoptimized prototype system. Under normal operating conditions the ionization fraction within the system is quite low, and so the propellant utilization is only a few tens of percent. One of the biggest challenges associated with plasma thrusters is the power sink represented by the plasma sheaths along the source tube boundaries, as discussed by Fruchtman. ${ }^{15}$ Possibilities to reduce these losses seem most likely to lie in an optimization of the applied magnetic field to aid plasma confinement as well as further optimization of the source cavity, as recently discussed by Chen. ${ }^{4}$

In summary, direct thrust measurements of a PM-HDLT have been performed using a pendulum thrust balance and a high sensitivity laser displacement system. Thrusts of between $0-3 \mathrm{mN}$ are measured for effective rf powers of 100$700 \mathrm{~W}$, which are in good agreement with calculations based on recent theoretical modeling.

${ }^{1}$ C. Charles and R. W. Boswell, Appl. Phys. Lett. 82, 1356 (2003).

${ }^{2}$ C. Charles, Plasma Sources Sci. Technol. 16, R1 (2007).

${ }^{3}$ C. Charles, J. Phys. D: Appl. Phys. 42, 163001 (2009).

${ }^{4}$ F. F. Chen, IEEE Trans. Plasma Sci. 36, 2095 (2008).

${ }^{5}$ K. Takahashi, K. Oguni, H. Yamada, and T. Fujiwara, Phys. Plasmas 15, 084501 (2008).

${ }^{6}$ D. M. Goebel and I. Katz, Fundamentals of Electric Propulsion: Ion and Hall Thrusters (Wiley, New Jersey, 2008).

${ }^{7}$ O. V. Batishchev, IEEE Trans. Plasma Sci. 37, 1563 (2009).

${ }^{8}$ J. Prager, T. Ziemba, R. Winglee, and B. Race Roberson, Phys. Plasmas 17, 013504 (2010).

${ }^{9}$ V. F. Virko, Y. V. Virko, V. M. Slobodyan, and K. P. Shamrai, Plasma Sources Sci. Technol. 19, 015004 (2010).

${ }^{10}$ C. Charles and R. W. Boswell, Appl. Phys. Lett. 91, 201505 (2007).

${ }^{11}$ M. D. West, C. Charles, and R. W. Boswell, J. Propul. Power 24, 134 (2008).

${ }^{12}$ K. Takahashi, C. Charles, R. W. Boswell, T. Kaneko, and R. Hatakeyama, Phys. Plasmas 14, 114503 (2007).

${ }^{13}$ C. Charles, R. W. Boswell, and M. A. Lieberman, Appl. Phys. Lett. 89, 261503 (2006).

${ }^{14}$ A. Fruchtman, Phys. Rev. Lett. 96, 065002 (2006).

${ }^{15}$ A. Fruchtman, IEEE Trans. Plasma Sci. 36, 403 (2008).

${ }^{16}$ T. E. Sheridan, Phys. Plasmas 7, 3084 (2000).

${ }^{17}$ T. Lafleur, C. Charles, and R. W. Boswell, J. Phys. D: Appl. Phys. 44 055202 (2011).

${ }^{18}$ K. Takahashi, Y. Itoh, and T. Fujiwara, J. Phys. D: Appl. Phys. 44, 015204 (2011).

${ }^{19}$ M. A. Lieberman and A. J. Lichtenberg, Principles of Plasma Discharges and Materials Processing, 2nd ed. (Wiley, New Jersey, 2005). 